\title{
Chemical Studies of Carcinoma
}

\section{A Toxic Substance Found in Gastric Carcinoma and its Effect on the Excised Frog Heart}

By

\author{
Kunihiro Maki \\ (槙 邦 弘) \\ From Prof. M. Muto's Surgical Clinic, Tohoku \\ University, Sendai
}

(Received for publication ,January 14, 1955)

It is generally accepted that a cancerous tissue contains some toxic substances, but the nature of these substances has not been elucidated, opinion on this matter being divided ${ }^{1 / 2)}$. Recently, Greenfield and Meister ${ }^{3 /}$ and Nakahara ${ }^{4}$ have reported the existence of a toxohormone in the dialyzate of the aqueous extract of a tumor tissue based on the results that the dialyzate lowered the catalase activity of the liver.

With a view of clarifying the nature of toxic substances contained in cancer, as a part of a series of studies being made by Assistant Professor K. Oh-Uti and his coworkers in our clinic, I investigated the effect of extracts of gastric carcinoma on the excised frog heart, and recognized their remarkable inhibiting action, which was not shown by the corresponding extracts of normal human gastric mucosa. By a fractionation study this inhibition has been traced to a substance in the portion of the extract which passed through the cellophane membrane on dialysis. So far as is known, the work to be reported here is the first one dealing with the inhibiting action of the gastric carcinoma extract on the frog heart.

\section{EXPERIMENTAL}

\section{Tests of the Gastric Carcinoma Extract}

The frog used was Rana nigromaculata, weighing 20 to $50 \mathrm{~g}$. The effect of an extract on an excised frog heart was recorded kymographically by the Shioya's third method at room temperature $\left(17\right.$ to $\left.27^{\circ} \mathrm{C}\right)$.

The extracts were prepared as follows: A sample of gastric carcinoma or normal gastric mucosa was taken from a specimen of gastric carcinoma or of gastric and duodenal ulcer excised in our clinic, minced, and washed well in water to remove blood constitutens and necrotic tissue. After removing moisture with filter paper as far as possible, $90 \mathrm{cc}$. of Ringer's solution for frogs (hereafter to be referred to simply as "Ringer's") or 
distilled water was added to $10 \mathrm{~g}$. of a tissue, and the mixture was kept at room temperature for 24 hours with constant shaking to facilitate the extraction, and then centrifuged. The supernatant thus obtained was used as the extract of that tissue. A fresh sample of gastric carcinoma or normal gastric mucosa was dried in vacuum to a mummy mass, to which was added distilled water or 2.5 per cent saline at the rate of $90 \mathrm{cc}$. per $10 \mathrm{~g}$. of the dried material. The mixture was then treated in a similar manner to obtain the extract. The concentration of each the extract was so adjusted that the extract contained $1 \mathrm{~g}$. of dry matter per $10 \mathrm{cc}$. by diluting the initial extract to a given extent estimated from the following previous test: A sample was taken from each extract, concentrated to a state of soup, and dried in vacuum to powder, which was weighed to determine the concentration of the extract. In experiments, a 100-fold dilution and its doubling dilutions were used.

1. Effect of the extract of a fresh tissue in Ringer's solution for frogs

The kymographic records obtained with varying dilutions of the extract of fresh gastric carcinoma in Ringer's are shown in Figure 1. At

Fig. 1. Effect of the extract of gastric carcinoma in Ringer's.

a. $1: 200$

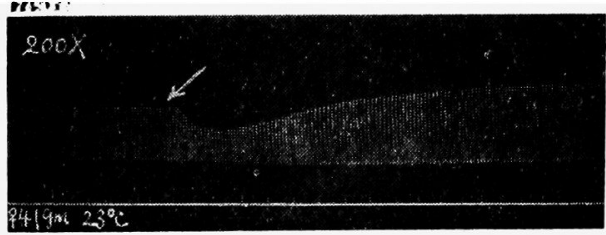

b. $1: 3200$

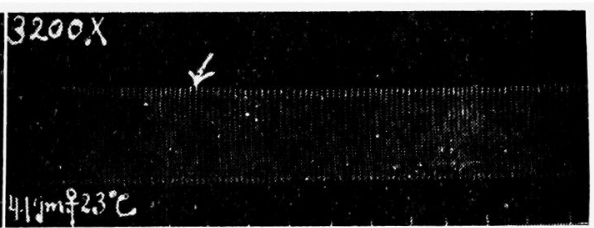

a dilution of $1: 200$ it will be seen that the inhibition was already remarkable immediately after exposure to the experimental solution, attained to its maximum after 12 seconds, then gradually decreased and almost disappeared after 42 seconds, a slight stimulatory effect being presented thereafter. The inhibition was perceptible also at dilutions up to $1: 800$, very weak at $1: 1600$, and not perceptible at $1: 3200$.

In the case of normal gastric mucosa (Fig. 2) the effect was very slightly inhibitory at $1: 200$ and $1: 400$, and rather stimulatory at $1: 800$.

Fig. 2. Effect of the extract of normal gastric mucosa in Ringer's.

a. $1: 200$

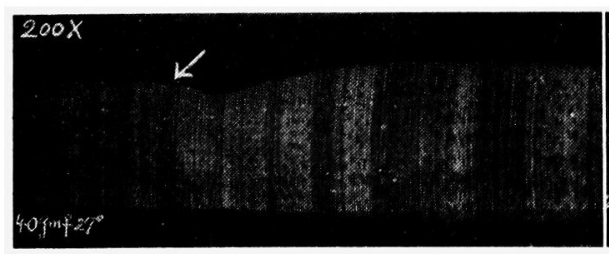

b. "क $1: 800$

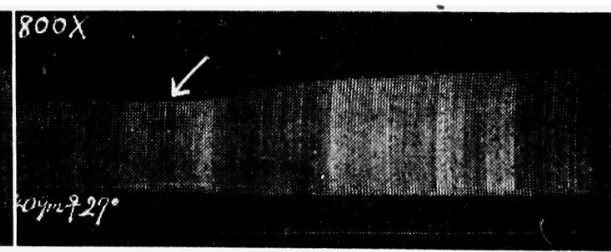


2. Effect of the extract of a fresh tissue in distilled water

Tests of the aqueous extract of fresh gastric carcinoma or normal gastric mucosa were performed with doubling dilutions (diluted with Ringer's) of a 100-fold dilution of the extract, in the same manner as before. In the cases of both the extract of gastric carcinoma and that of normal gastric mucosa, the effect was remarkably inhibitory at 1:100 and $1: 200$; though the recovery of the heart began after 30 to 40 minutes, it was quite irregular, different from that in the case of an extract in Ringer's. As shown in Table I, the inhibition decreased with the increase of dilution

TABLE I

Effect of the Extracts Mummy-dried Materials in Distilled Water*

\begin{tabular}{c|c|c|c|c|c}
\hline $1:$ & 100 & 200 & 400 & 800 & 1600 \\
\hline Gastric carc. & $H$ & $H$ & + & + & - \\
Normal gastric mucosa & $H$ & $H$ & - & - & -
\end{tabular}

$H, \mathrm{D}=0-15 \% ; H, 16-50 \% \dagger+, 31-95 \% ; \pm$, higher than $96 \%$. D (reciprocal of degree of inhibition) $=$

Minimum amplitude of heat beat Normal amplitude

in both extracts, but more rapidly in the case of normal gastric mucosa. The dilution at which the inhibition first became negative was 1:400 with normal gastric mucosa, and as high as $1: 1600$ with gastric carcinoma.

3. Effect of the extract of a dried tissue in distilled water

The results obtained with the aqueous extracts of dried gastric carcinoma and normal gastric mucosa are summarized in Table II, where it will be seen that the inhibition was presented by the extracts of both

TABLE II

Effect of the Extracts Mummy-dried Materials in Distilled Water*

\begin{tabular}{l|c|c|c|c|c}
\hline 1: & 100 & 200 & 400 & 800 & 1600 \\
\cline { 2 - 5 } Gastric. & $H$ & H & + & + & - \\
Normal gastric mucosa & $H$ & $H$ & - & - & -
\end{tabular}

* Explanation for signs, in Table I.

materials, but the gastric carcinoma extract was about 4 times as inhibitory as the normal gastric mucosa extract.

4. Effect of the extract of a dried tissue in 2.5 per cent saline

Similar tests with the extracts in 2.5 per cent saline instead of distilled water yielded the results quite similar to those with the aqueous extracts, as 
shown in Table III.

TABLE III

Effect of the Extracts Mummy-dried Materials in $2.5 \%$ Saline

\begin{tabular}{c|c|c|c|c|c}
\hline 1: & 100 & 200 & 400 & 800 & 1600 \\
\hline Gastric carc. & $H$ & + & + & \pm & - \\
Normal gastric mucosa & + & + & - & - & -
\end{tabular}

5. Differences in the intensity of heart inhibition according to the form of carcinoma

The intensity of heart inhibition of gastric carcinoma extracts was found to differ according to the form of the carcinoma, for example, the highest dilution at which the inhibition was still perceptible varied from $1: 800$ to $1: 20000$ as shown in Table IV,carcinoma solidum gelatinosum and carcinoma adenomatosum gelatinosum presumably being the most effective two.

TABLE IV

Differences in the Intensity of Heart Inhibition according to the Form of Carcinoma

\begin{tabular}{c|c|c}
\hline Case & Highest dilution* $^{*}$ & \multicolumn{1}{|c}{ Form of carcinoma } \\
\cline { 1 - 2 } & 800 & Carc. adenomatosum simplex \\
2 & 1600 & Carc. solidum diffusum \\
3 & 1600 & Carc. solidum diffusum \\
4 & 5000 & Carc. solidum alveolare \\
5 & 6000 & Carc. solidum gelatinosum \\
6 & 20000 & Carc. adenomatosum gelatinosum \\
& * Highest dilution at which an inhibition of degree (see Table I) is perceptible.
\end{tabular}

Summarizing the results of the experiments described in this section it will be concluded that all kinds of extracts of gastric carcinoma, fresh and dried, presented nearly the same order of heart-inhibiting effect, which was about 4 times or more as strong as that of normal gastric mucosa.

\section{Heart-Inhibiting Effect of the Dialyzate of a Crude Extract}

With a view to tracing the heart-inhibiting substance in the crude extracts of gastric carcinoma, the extracts of fresh gastric carcinoma and normal gastric mucosa (10 g. in each case) in Ringer's, 2.5 per cent saline, and distilled water were dialyzed across the cellophane membrane for 48 
hours, and the dialyzates and the dialyzed extracts were tested for the inhibiting effect.

1. Tests of the dialyzed extracts

In the cases of the extracts in Ringer's and 2.5 per cent saline, each dialyzed extract was centrifuged to remove the preciptitate, and the supernatant was tested for the heart-inhibiting effect at varying dilutions, with the results that neither the gastric carcinoma extract nor the normal gastric mucosa extract showed inhibition, and indeed the effect of the former was stimulatory, not inhibitory, at a dilution of $1: 250$ (Fig. 3 a.). When these

Fig. 3. Effect of a gastric carcinoma extract, dialyzed against Ringer's,
a) supernatant, $1: 250$.
b) sediment rinsings, $1: 1000$.
c) alkalisoluble sediment, $1: 250$.

a.

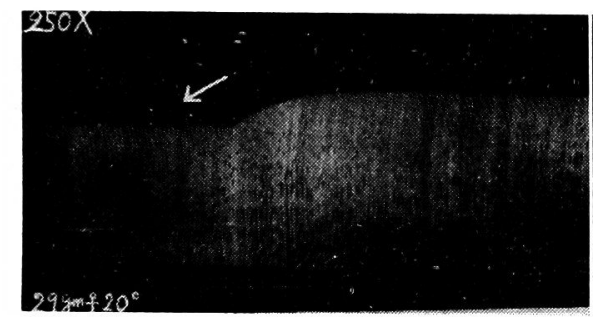

c.

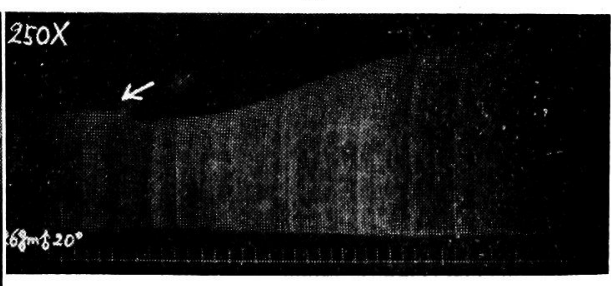

b.

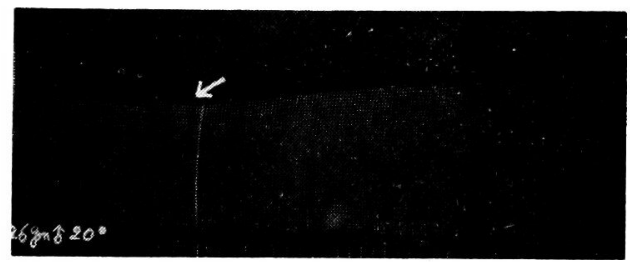

supernatants were condensed and tested, there were often found heart stimulation but never inhibition.

Next, the sediment obtained by the above centrifugation of each dialyzed extract was rinsed repeatedly with distilled water, and the rinsings were made into the isotonic Ringer's and tested for the effect on the heart, with the results that a slight stimulation, not inhibition, was shown (Fig. 3. b.). Furthermore, Ringer's was added to the sediment, and the $\mathrm{pH}$ was adjusted to 7.6 with $\mathrm{N} / 10$ sodium hydroxide to dissolve the sediment. The solution was then centrifuged, and the supernatant, which was alkalisoluble, was tested. In this case there appeared a slight inhibition (Fig. 3. c.). The extracts of normal gastric mucosa were treated in a similar manner, but showed no inhibition.

Summarizing the results described in this section, it may be concluded that each extract of gastric carcinoma and normal gastric mucosa lost its 
inhibiting action after the dialysis.

\section{Tests of the dialyzates}

In testing the dialyzates, only the aqueous extracts of gastric carcinoma and normal gastric mucosa were used to exclude a possible effect of the salts of the solution themselves against which dialysis was done. Thus, an extract obtained when $90 \mathrm{cc}$. of distilled water was added to $10 \mathrm{~g}$. of a tissue was dialyzed and the dialyzate was collected. The sac containing the extract was then immersed in new distilled water for further dialysis and after a given period the second dialyzate was collected. By repeating this many times a total of 800 to $1000 \mathrm{cc}$. of the dialyzate was obtained. This was condensed in vacuum so as to contain solid matter 1 per cent (i.e., 1:100 dilution), and was tested for the effect on the heart at its doubling dilutions (diluted with Ringer's), with the results shown in Figure 4 , where it will be seen that there were presented remarkable inhibition at $1: 200$ to $1: 800$, slight inhibition at $1: 1600$ and $1: 3200$, and no inhibition at $1: 6400$. As for the dialyzate of normal gastric mucosa there were presented remarkable inhibition at $1: 200$ and $1: 400$, slight inhibition at $1: 800$, and no inhibition at $1: 1600$, as shown in Figure 5. Thus it may be seen that the dialyzate of a gastric carcinoma extract presented an inhibition 2 to 4 times as strong as the inhibition of normal gastric mucosa.

Fig. 4. Effect of the dialyzate of a gastric carcinoma extract.

a. $1: 200$

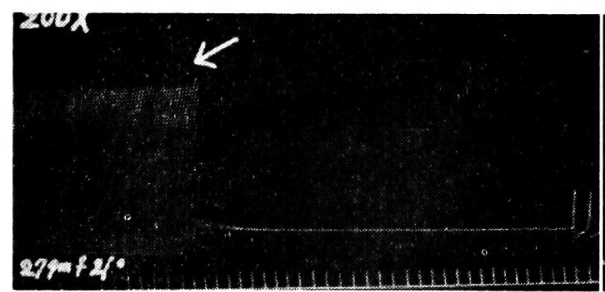

b. $1: 6400$

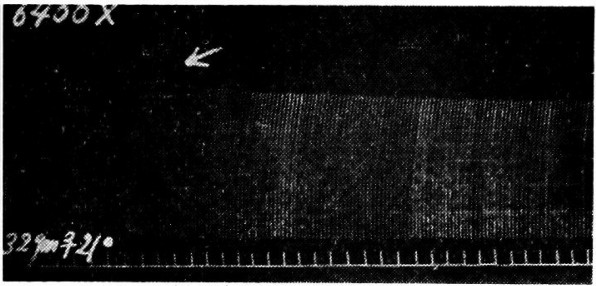

Fig. 5. Effect of the dialyzate of a normal gastric mucosa extract.

a. $1: 200$

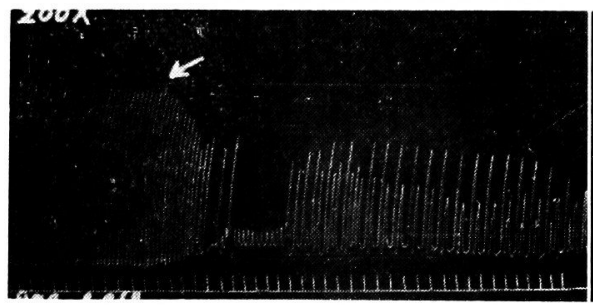

b. $1: 1600$

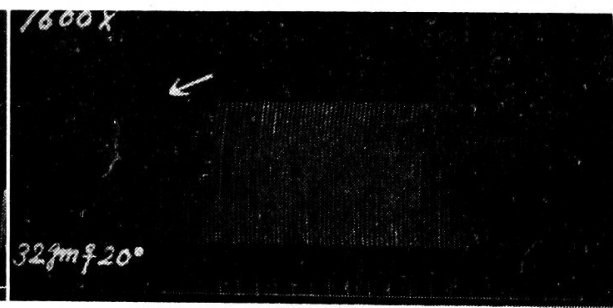

Tracing of the Heart-inhibiting Substance

As the presence of some heart-inhibiting substance in the dialyzate 
of an extract was demonstrated, the next step was to examine the nature of this substance by submitting it to various chemical treatments and testing the products for the effect on the heart.

\section{Effect of dryind}

After being tested for the heart inhibition, the dialyzate of a gastric carcinoma extract was condensed to a syrup, and dried in vacuum. Not a perfectly dry but a viscous lipoid substance was obtained. To $20 \mathrm{mg}$. of this dried material was added $2 \mathrm{cc}$. of Ringer's to make a 1:100 dilution, and the solution was tested for the effect on the heart at doubling dilutions (diluted with Ringer's), with the results shown in Table V, where it will be seen that drying exerted no influence on the intensity of heart inhibition.

\section{TABLE V}

Effect of Drying on the Intensity of Heat Inhibition

(Material: Gastric Carcinoma, dried; dialyzate)

\begin{tabular}{c|c|c|c|c|c}
\hline $1:$ & 200 & 500 & 1000 & 2000 & 4000 \\
\cline { 2 - 3 } Before drying & $\#$ & + & + & + & - \\
After drying & $\ldots$ & + & + & + & -
\end{tabular}

\section{Effect of heating}

With $10 \mathrm{mg}$. of the above dried material a $1: 700$ dilution was prepared. Some of the solution was used for testing for the inhibition. The remaining solution was heated for 10 minutes at $80^{\circ} \mathrm{C}$. or $100^{\circ} \mathrm{C}$., and tested at varying dilutions. However, there were found no differences in their effect between the materials before and after the heating as shown in Figure 6.

Fig. 6. Effect of the dialyzate of a gastric carcinoma extract, $1: 700$, $80^{\circ} \mathrm{C}$., 10 minutes heating.

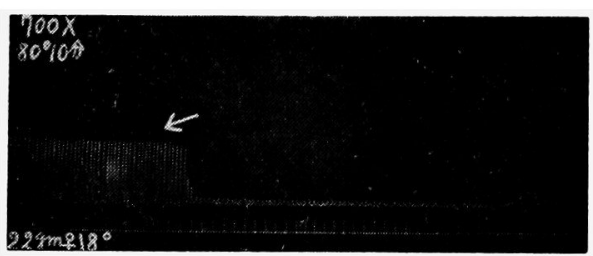

\section{Effect of treating with a strong acid}

To $10 \mathrm{mg}$. of the dried material was added 2 cc. of Ringer's to make a $1: 200$ dilution, to which was added 3 drops of $\mathrm{N} / 10$ hydrochloric acid. After the solution was let to stand for 15 minutes, the $\mathrm{pH}$ was adjusted to 7.0 with $\mathrm{N} / 10$ sodium hydroxide. Tests with this solution yielded the results shown in Table VI that the treatment with the acid brought about 


\section{TAB LE VI}

Effect of a Strong Acid on the Intensity of Heart Inhibition (Material: gastric carcinoma, dried; dialyzate)

\begin{tabular}{c|c|c|c|c|c}
\hline $1:$ & 200 & 400 & 800 & 1600 & 3200 \\
\cline { 2 - 5 } & $H$ & + & \pm & - & - \\
Strong acid & $+\#$ & + & + & + & -
\end{tabular}

* Doubling dilutions of a $1: 100$ dilution of the dialyzate of the extract of dried material.

a slight decrease in the intensity of heart inhibition.

4. Effect of treating with the ion exchange resin

a) Cations. An amount of $70 \mathrm{mg}$. of the dried material was dissolved in 2 cc. of distilled water, to which was added $5 \mathrm{~g}$. (wet weight) of cations (Amberlite/I R 120). The mixture was shaken for 20 minutes. To the supernatant was added rinsings that had been obtained by rinsing ion exchange resin 3 times with $5 \mathrm{cc}$. of distilled water. After the $\mathrm{pH}$ had been adjusted to 7.0, the solution was condensed in vacuum to dryness. The dried material thus obtained was tested at varying dilutions, with the results, as shown in Fig. 7, that the inhibition was slight at $1: 25$ and $1: 50$, and negative at $1: 200$.

b) Anions. An amount of $70 \mathrm{mg}$. of the dried material was treated with $10 \mathrm{~g}$. (wet weight) of anions (Amberlite/IRA 400) in the same manner as before. After the treatment, the material showed a strong inhibiting effect, the effect being perceptible even at $1: 900$ (Fig. 8).

Fig. 7. Effect of the dialyzate of gastric carcinoma, passed through cations.

a. $1: 25$

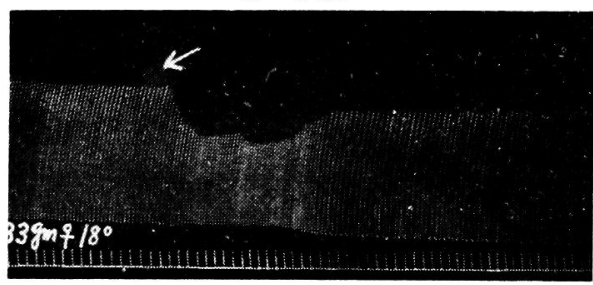

b. $1: 200$

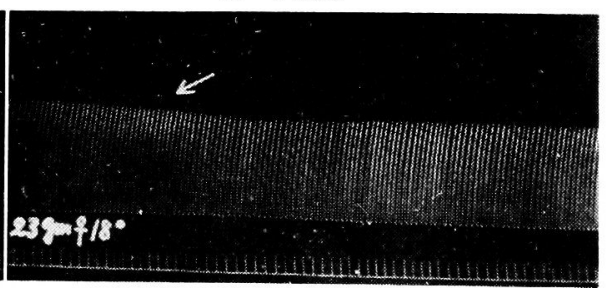

c) Cations and anions. When the material was treated first with cations, then with anions each for 30 minutes in the same manner as before, it showed no inhibiting action even at $1: 30$ (Fig. 9).

From these results it may be concluded that the heart-inhibiting substance was absorbed mainly by cation exchange resin, and when passed through both ion exchange resins the substance was absorbed completely. 
Fig. 8

Fig. 9

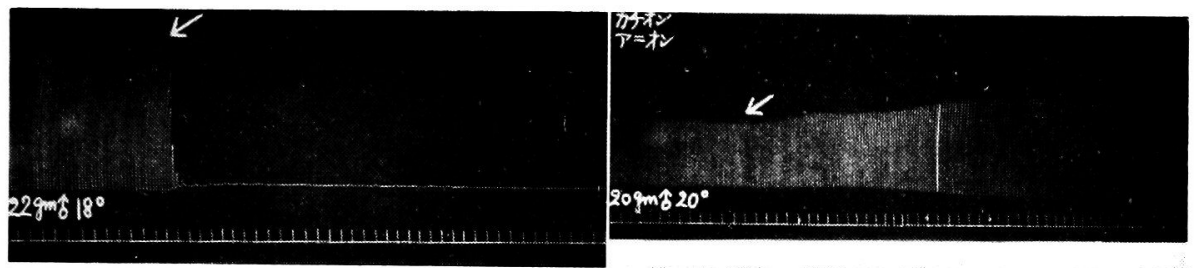

Fig. 8. Effect of the dialyzate of gastric carcinoma, passed through anions. $1: 25$.

Fig. 9. Effect of the dialyzate of gastric carcinoma, passed through cations and anions, $1: 30$.

5. Effect of treating with organic solvents

a) Alcohol. i. 70 per cent alcohol, room temperature. To an amount of $40 \mathrm{mg}$. of the dried material was added $20 \mathrm{cc}$. of alcohol, and the mixture was agitated for 30 minutes and centrifuged. The sediment was repeatedly rinsed with alcohol, and the rinsings were united with the supernatant. The solution was condensed in vacuum to dryness, redissolved in Ringer's. The above sediment was dried in vacuum, and redissolved in Ringer's. The two preparations thus obtained were tested for the effect on the heart and were found to show nearly equal degrees of inhibition at the same concentration.

ii. 99 per cent alcohol, room temperature. To an amount of 20 mg. of the dried material was added $20 \mathrm{cc}$. of 99 per cent alcohol, and the mixture was agitated for 30 minutes, and then centrifuged for 10 minutes at $3000 \mathrm{rpm}$. The supernatant was dried in vacuum to powder. This powder was tested at varying dilutions, and it was found (Table VII) that the material had a remarkable inhibition on the heart, the effect disappearing first at $1: 1600$.

iii. 99 per cent alcohol, boiled. To an amount of $20 \mathrm{mg}$. of the dried material was added $50 \mathrm{cc}$. of 99 per cent alcohol, and the mixture was agitated for 10 minutes, boiled for 10 minutes at $100^{\circ} \mathrm{C}$., and filtered quickly. The filtrate was condensed in vacuum, and redissolved in $2 \mathrm{cc}$. of Ringer's to make a $1: 100$ dilution. The inhibiting action of this preparation was so strong as to be still fairly manifest at $1: 1600$.

b) Ether. The inhibiting substance was found not to dissolve in ether (Table VII).

c) Chloroform. The inhibiting substance was found to dissolve well in dehydrated chloroform (Table VII).

d) Benzole and acetone. The inhibiting substance slightly dissolved in benzole, but not in acetone.

Summarizing all these results given in tihs section, it may be said 
TABLE VII

Effect of Treatment with Organic Solvents

(Material : gastric carcinoma, dried; dialyzate)

\begin{tabular}{|c|c|c|c|c|c|c|}
\hline 1: & 100 & 200 & 400 & 800 & 1600 & 3200 \\
\hline $99 \%$ Alcohol $\int_{\text {temperature }}^{\text {room }}$ & \# & H & + & \pm & - & - \\
\hline boiled & \# & H & + & + & + & - \\
\hline Ether & - & - & - & - & - & - \\
\hline Chloroform & \# & H & H & + & - & - \\
\hline Benzene & + & \pm & - & - & - & - \\
\hline Acetone & - & - & - & - & - & - \\
\hline Control & \# & \# & H & + & + & - \\
\hline
\end{tabular}

Control: see Table VI.

TABLE VIII

Qualitative Tests of Fractions of the Heart-inhibiting Sustance

\begin{tabular}{|c|c|c|c|c|c|}
\hline & & $\begin{array}{l}\text { Biuret } \\
\text { reaction }\end{array}$ & $\begin{array}{l}\text { Molisch } \\
\text { reaction }\end{array}$ & $\begin{array}{l}\text { Bial } \\
\text { reaction }\end{array}$ & $\begin{array}{l}\text { Dische } \\
\text { reaction }\end{array}$ \\
\hline 1) & $\begin{array}{l}\text { Gastric carc. } \\
\text { Normal gastric mucosa }\end{array}$ & H & 世 & H & + \\
\hline 2) & $\begin{array}{l}\text { Gastric carc. } \\
\text { Normal gastric mucosa }\end{array}$ & + & $H$ & $\begin{array}{l}\text { 姍 } \\
\text { H }\end{array}$ & + \\
\hline 3) & $\begin{array}{l}\text { Gastric carc. } \\
\text { Normal gastric mucosa }\end{array}$ & $\begin{array}{l}+ \\
+\end{array}$ & $\begin{array}{l}+ \\
+\end{array}$ & $\begin{array}{l}+ \\
+\end{array}$ & - \\
\hline 4) & $\begin{array}{l}\text { Gastric carc. } \\
\text { Normal gastric mucosa }\end{array}$ & $\overline{-}$ & $\overrightarrow{-}$ & $\overline{-}$ & $\overline{-}$ \\
\hline 5) & $\begin{array}{l}\text { Gastric carc. } \\
\text { Normal gastric mucosa }\end{array}$ & - & $\begin{array}{l} \pm \\
\pm\end{array}$ & $\overline{-}$ & - \\
\hline
\end{tabular}

that the inhibiting substance in the dialyzate of an extract is not affected by drying to powder, slightly weakened by a strong acid, absorbed by ion exchange cation, and easily soluble in alcohol and chloroform.

\section{Qualitative Tests of Fractions of the Heart-inhibiting Substance}

Biuret, Molisch's, Bial's, and Dische's tests of gastric carcinoma and normal gastric mucosa were performed on their 1) aqueous extracts, 2) dried materials of the dialyzates, 3) extracts in 99 per cent alcohol, 4) extracts by boiling in 99 per cent alcohol, and 5) extracts in chloroform (extracted twice), with the results shown in Table VIII. All the solutions 
were tested at a dilution of $1: 100$. It will be seen that between gastric carcinoma and normal gastric mucosa there were no significant differences in the four qualitative tests of both extracts and dialyzates, and that the alcohol and chloroform solutions of the heart-inhibiting substance yielded only very weak or no positive results in the four tests. From these it may be assumed that the heart-inhibiting substance are negative to these qualitative tests. In addition, tests for hemolysis and phosphorus were made on the extracts by boiling in 99 per cent alcohol, which presented the most remarkable inhibition, but with negative results.

\section{Summary}

1. The extract of gastric carcinoma in distilled water, Ringer's solution for frogs or 2.5 per cent saline presented an inhibiting effect on the excised frog heart more than four times as strong as that of the extract of normal human gastric mucosa when studied by the kymographic method.

2. When the extract was dialyzed across the cellophane membrane for 48 hours the heart-inhibiting substance was demonstrated in the dialyzate, not in the dialyzed extract.

3. Various chemical and physical treatments were submitted to this substance to study its nature, and it was found that the inhibiting substance was absorbed by cation exchange resin, and soluble in alcohol and chloroform.

In conclusion, I wish to express my sincere gratitude to Assistant Professor K. Ishizuka of the Pharmacological Institute and to Dr. H. Kawasaki of the Medicochemical Institute of Tohoku University.

\section{References}

1) Takamura, Nagasaki Igakkai Zasshi, 1942, 20, 1296.

2) Harima, Tohoku Igaku Zasshi, 1954, 50, 581.

3) Greenfield \& Meister, J. Nat. Cancer Inst., 1951, 2, 997.

4) Nakahara \& Fukuoka, Gann, 1954, 45, 67. 